\title{
LA-UR-98-
}

Title:

\section{OPTICAL NONLINEARITIES AND ULTRAFAST CARRIER DYNAMICS IN SEMICONDUCTOR QUANTUM DOTS}

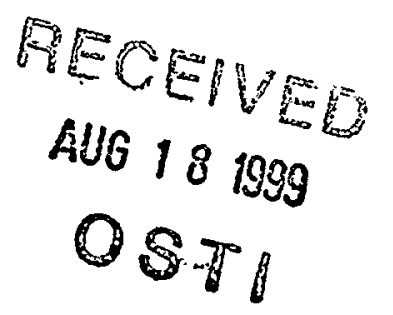

V. Klimov, CST-6

Submitted to:

D. McBranch, CST-6

C. Schwarz, CST-6

Los Alamos National Laboratory

Los Alamos, NM 87545

Proceedings of the O.S. A. Conference

"Nonlinear Opitcs '98"

Princeville, August 10-14, 1998

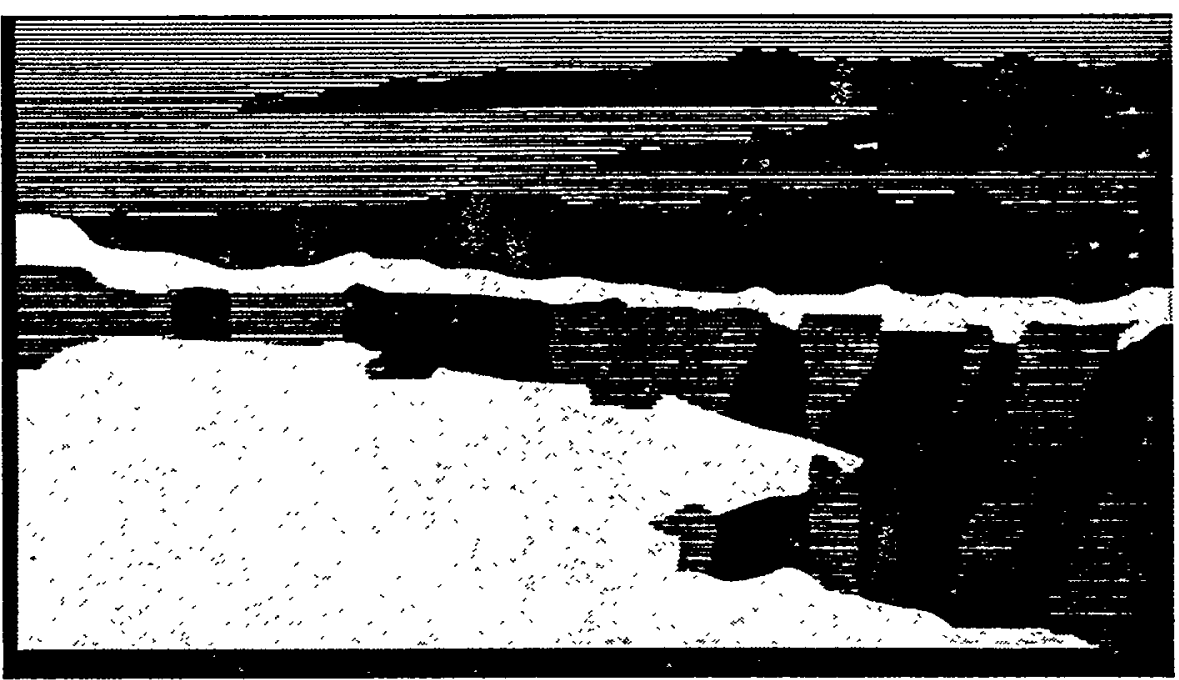

Los Alamos National Laboratory, an aftimative action/equal opportunity employer, is operated by the University of Califomia for the U.S. Department of Energy under contract W-7405-ENG-36. By acceptance of this article, the publisher recognizes that the U.S. Government retalis a nonexclusive, royalty-free icense to publish or reproduce the published form of this contribution, or to allow others to do so, for U.S. Govemment purposes. The Los Alamos National Laboratory requests that the publisher identify this article as work performed under the auspices of the U.S. Department of Energy. 


\section{DISCLAIMER}

This report was prepared as an account of work sponsored by an agency of the United States Government. Neither the United States Government nor any agency thereof, nor any of their employees, make any warranty, express or implied, or assumes any legal liability or responsibility for the accuracy, completeness, or usefulness of any information, apparatus, product, or process disclosed, or represents that its use would not infringe privately owned rights. Reference herein to any specific commercial product, process, or service by trade name, trademark, manufacturer, or otherwise does not necessarily constitute or imply its endorsement, recommendation, or favoring by the United States Government or any agency thereof. The views and opinions of authors expressed herein do not necessarily state or reflect those of the United States Government or any agency thereof. 


\section{DISCLAIMER}

Portions of this document may be illegible in electronic image products. Images are produced from the best available original document. 


\title{
Optical Nonlinearities and Ultrafast Carrier Dynamics in Semiconductor Quantum Dots
}

\author{
V. Klimov, D. McBranch, and C. Schwarz \\ Chemical Sciences and Technology Division, CST-6, MS-J585, \\ Los Alamos National Laboratory, Los Alamos, NM 87545
}

Low-dimensional semiconductors have attracted great interest due to the potential for tailoring their linear and nonlinear optical properties over a wide-range. Semiconductor nanocrystals (NC's) represent a class of quasi-zero-dimensional objects or quantum dots. Due to quantum confinement and a large surface-to-volume ratio, the linear and nonlinear optical properties, and the carrier dynamics in NC's are significantly different from those in bulk materials [1, 2]. Trapping at surface states can lead to a fast depopulation of quantized states, accompanied by charge separation and generation of local fields which significantly modifies the nonlinear optical response in NC's [3]. 3D carrier confinement also has a drastic effect on the energy relaxation dynamics [2]. In strongly confined NC's, the energy-level spacing can greatly exceed typical phonon energies. This has been expected to significantly inhibit phonon-related mechanisms for energy losses, an effect referred to as a phonon bottleneck [4]. It has been suggested recently that the phonon bottleneck in 3D-confined systems can be removed due to enhanced role of Auger-type interactions [5]. In this paper we report femtosecond (fs) studies of ultrafast optical nonlinearities, and energy relaxation and trapping dynamics in three types of quantum-dot systems: semiconductor $\mathrm{NC} /$ glass composites made by high temperature precipitation, ion-implanted NC's, and colloidal NC's. Comparison of ultrafast data for different samples allows us to separate effects being intrinsic to quantum dots from those related to lattice imperfections and interface properties.

High-sensitivity fs nonlinear transmission experiments in the novel chirp-free configuration [6] were applied to measure pump-induced absorption changes for optical transitions involving the lowest (1S) and the first excited (1P) electron states $\left(B_{1}\right.$ and $B_{3}$ features in Fig. 1, respectively) in CdSe NCs of different radii, with 1S-1P energy separation up to 16 longitudinal optical phonon energies. At early times after excitation, the nonlinear optical response of the NC's is dominated by two effects: state-filling induced bleaching of optical transitions [2] $\left(\mathrm{B}_{1}-\mathrm{B}_{3}\right.$, Fig. 1) and the Coulomb two-pair interaction (biexciton effect) [7]. While state-filling affects only transitions coupling occupied states, the Coulomb interaction influences all transitions, resulting in transition shifts which are seen as derivative-like features in transient absorption (TA) (see feature at $\sim 1.85 \mathrm{eV}$ in the early time TA spectra in Fig. 1). Analysis of the TA allows us to extract the state-filling-induced portion of the nonlinear signal, which provides the information on population/depopulation rates of the adjacent (1S and 1P) electronic states. Instead of the drastic reduction of the energy relaxation rate expected due to a phonon bottleneck [4], we observe a fast sub-ps 1P-to-1S relaxation (inset to Fig. 1), indicating the opening of new relaxation channels which dominate any phonon bottleneck effects. We observe the enhancement in the relaxation rate in NCs of smaller radius, which strongly suggests that energy relaxation is mediated by nonlinear Auger-type processes. Similar energy relaxation behavior is observed in different types of $\mathrm{NCs}$, indicating that ultrafast electron relaxation, not limited by a phonon bottleneck, is a general feature of quantum-dot systems. 


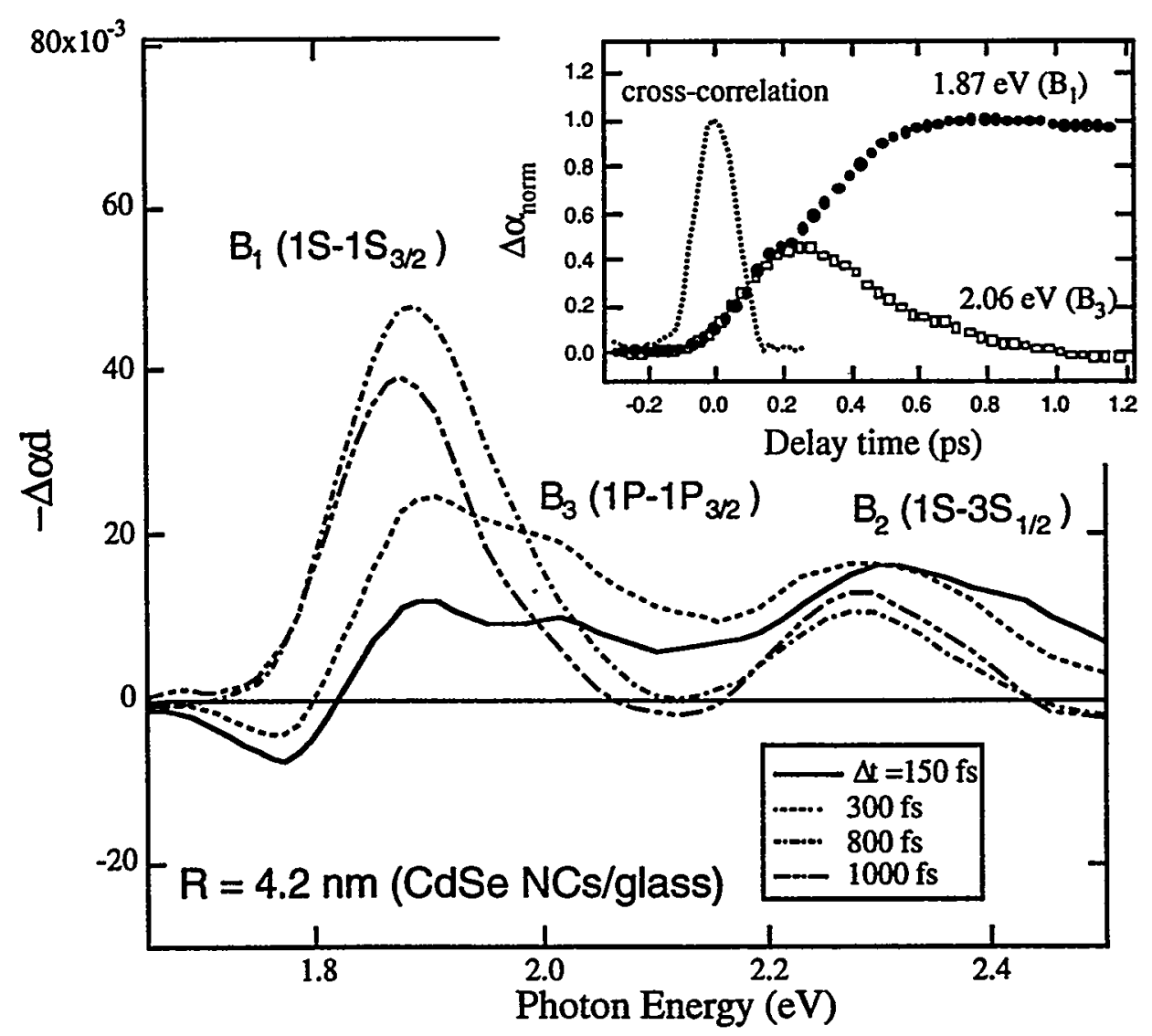

Figure 1. Chirp-free TA spectra recorded at different delay times after excitation for CdSe NC/glass sample with NC mean radius of $4.2 \mathrm{~nm}$. Inset: normalized 1S (circles) and IP (squares) dynamics indicating the 1P-to-1S relaxation time of $\sim 300 \mathrm{fs}$.

In contrast to the initial energy relaxation, the subsequent 1S-electron decay is strongly sample dependent. In colloidal samples with passivated surfaces, the depopulation of the 1S state is dominated by radiative recombination, whereas in glass samples made by both high-temperature precipitation and ion implantation, the 1S electrons relax via efficient ps trapping at deep defect states, an effect which is strongly enhanced in NCs of smaller radius (Fig. 2). In the inset to Fig. 2, we show that the size dependence of the 1S relaxation time follows an $\mathrm{R}^{6}$ dependence. This can be explained in terms of confinement-induced squeezing of the 1S electron wave function, resulting in an increased overlap with the wave function of the deep-trap state. Application of fs up-conversion photoluminescence and TA allowed us to separate electron and hole relaxation paths [8]. We observed very fast PL decay (attributed to sub-ps hole trapping). The slower decay of the TA signal is controlled by depopulation of electron states which occurs on the 1-10 ps time scale.

Studies of pump intensity-dependent TA indicate a change in carrier relaxation channels at high pump levels. This is manifested as a distinct difference in the nonlinear optical response measured at low and high pump intensities in quasiequilibrium at long times after excitation. Analysis of the spectral- and time-dependence of the nonlinear transmission over 
a wide pump intensity range indicates that this difference is due to carrier trapping at surface states activated by the Auger process. Surface trapping leads to efficient charge separation, with associated local electric fields [3], drastically modifying the nonlinear optical response

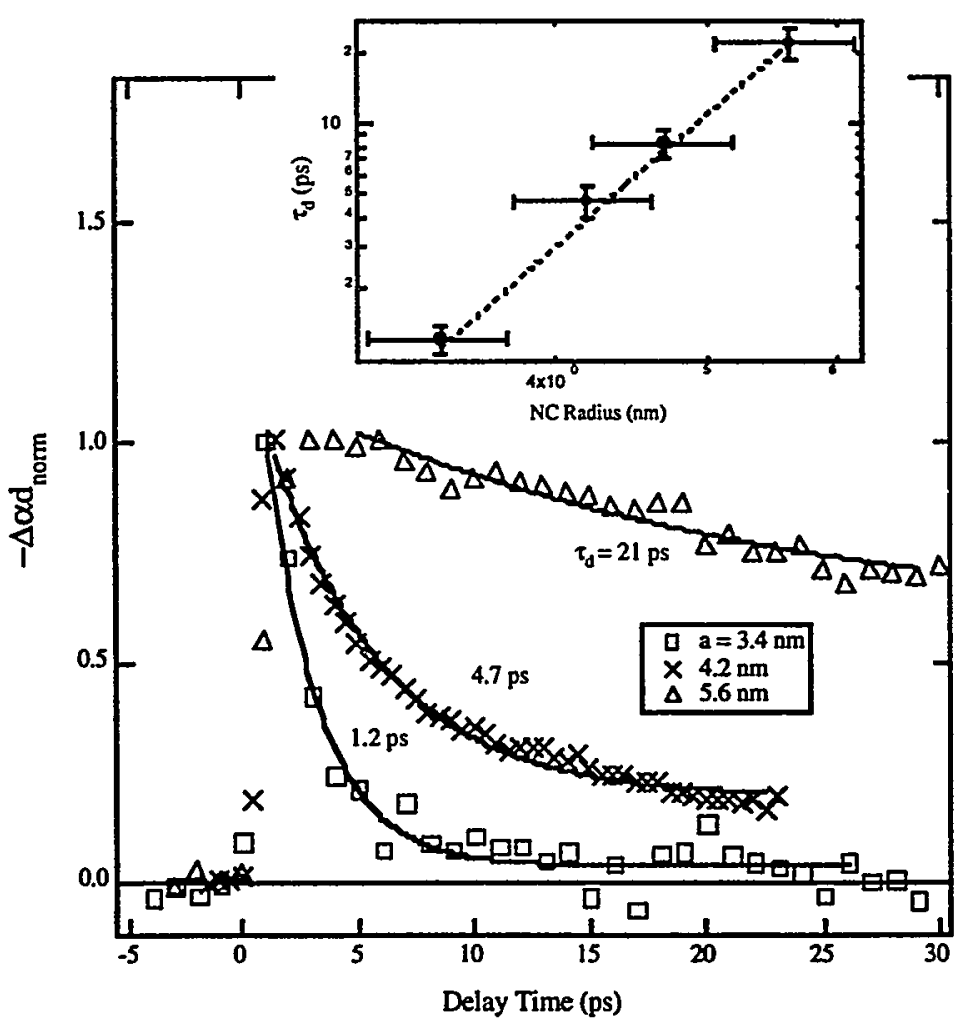

Figure 2. 1S-bleaching dynamics in CdSe/glass samples with $\mathrm{NC}$ mean radii $\mathrm{R}$ $=3.4$ (open squares), 4.2 (crosses), and $5.6 \mathrm{~nm}$ (open triangles). Inset: size dependence of the $1 \mathrm{~S}$ relaxation time (symbols), fit to an $\mathrm{R}^{6}$ dependence (line).

of NC's at high pump fluences. Using NCs in different types of matrices, we studied the effect of the height of the confinement barrier on the efficiency of Auger-assisted surface trapping.

[1] A. P. Alivasatos, Science 271, 933 (1996).

[2] V. Klimov, in Handbook on Nanostructured Materials and Nanotechnology, edited by H. Nalwa (to be published by Academic Press, 1998).

[3] V. Klimov and D. McBranch, Phys. Rev. B 55, 13173 (1997).

[4] U. Bockelman and G. Bastard, Phys. Rev. B 42, 8947 (1990).

[5] A. L. Efros, V. A. Kharchenko, and M. Rosen, Solid State Commun. 93, 281 (1995).

[6] V. Klimov and D. McBranch, Opt. Lett. 23, (February, 1998).

[7] V. Klimov, S. Hunsche, and H. Kurz, Phys. Rev. B 50, 8110 (1994).

[8] V. Klimov, P. Haring Bolivar, and H. Kurz, Phys. Rev. B 53, 1463 (1996). 